\title{
OPEN Nonresonant amplification of spin waves through interface magnetoelectric effect and spin-transfer torque
}

\author{
Piotr Graczyk ${ }^{1 \bowtie}$ \& Maciej Krawczyk ${ }^{2}$
}

We present a new mechanism for manipulation of the spin-wave amplitude through the use of the dynamic charge-mediated magnetoelectric effect in ultrathin multilayers composed of dielectric thinfilm capacitors separated by a ferromagnetic bilayer. Propagating spin waves can be amplified and attenuated with rising and decreasing slopes of the oscillating voltage, respectively, locally applied to the sample. The way the spin accumulation is generated makes the interaction of the spin-transfer torque with the magnetization dynamics mode-selective and restricted to some range of spin-wave frequencies, which is contrary to known types of the spin-transfer torque effects. The interfacial nature of spin-dependent screening allows to reduce the thickness of the fixed magnetization layer to a few nanometers, thus the proposed effect significantly contributes toward realization of the magnonic devices and also miniaturization of the spintronic devices.

Spin waves are potential candidates to replace electrons in logical systems to operate faster, with ultralow energy consumption and operating at sub-micrometer scale. Much effort has been devoted to develop methods of spinwave generation, steering and conversion which led to the first laboratory realization of the spin-wave-based logic units $^{1}$. However, the main challenge for the magnon computing, which is relatively high attenuation, remains unresolved. Although spin waves have usually higher propagation lengths than spin currents, they are still far behind electric signals.

We can split the methods of control of spin-wave attenuation into two categories. The first relies on resonant or parametric amplification by means of microwaves ${ }^{2-5}$ or magnetoelastic fields ${ }^{6-10}$ and thus it enhances the signal at a particular frequency. The second method relies on the exchange interaction between spin-polarized conductive electrons and localized magnetic moments through spin-transfer torque (STT). For this method the spin accumulation has to be generated in the system by means of spin-dependent conductivity ${ }^{11,12}$, spindependent surface screening ${ }^{13}$ or various other effects related to the spin-orbit coupling: spin Hall effect ${ }^{14-18}$, Rashba-Edelstein effect ${ }^{19}$, generalized spin-orbit torques ${ }^{20-22}$ and topological effects ${ }^{23,24}$.

STT is regarded to be non-selective, i.e., it affects all the spin-wave modes in the system regardless of the frequency or the wavevector ${ }^{5}$. It means, that the transmitted signal is enhanced or suppressed together with the incoherent noise. While it is correct statement for the spin current generated by the spin Hall effects (where the polarization of nonequilibrium spins is determined by the direction of the electric field in heavy metal and the geometry but not the direction of magnetic moment in the adjacent ferromagnet), it does not necessarily apply to other mechanisms of spin current generation. Here, we propose another approach and demonstrate, that the spin accumulation driven by the spin-dependent surface screening in ultrathin magnetoelectric laminate works differently: it effectively amplifies and modulates only coherent spin wave modes of specific symmetry and group velocity.

In our proposition, the modulation of spin-wave amplitude takes place in the magnetoelectric cell (MEC, Fig. 1) which consists of two high-permittivity dielectric (D) nanocapacitors in series connected through a conductive ferromagnetic bilayer. The thin ferromagnetic layers, made from Fe and Co are separated by a nonmagnetic metal $(\mathrm{Cu})$.

The principle of operation is as follows (see, Fig. 1). The ac voltage applied to the MEC generates time-varying screening charges at the ferromagnetic interfaces with dielectrics. Because the density of the screening charge is

\footnotetext{
${ }^{1}$ Institute of Molecular Physics, Polish Academy of Sciences, M. Smoluchowskiego 17, 60-179 Poznan, Poland. ${ }^{2}$ Institute of Physics and Quantum Information, Faculty of Physics, Adam Mickiewicz University in Poznan, Uniwersytetu Poznańskiego 2, 61-614 Poznan, Poland. ${ }^{\circledR}$ email: graczyk@ifmpan.poznan.pl
} 


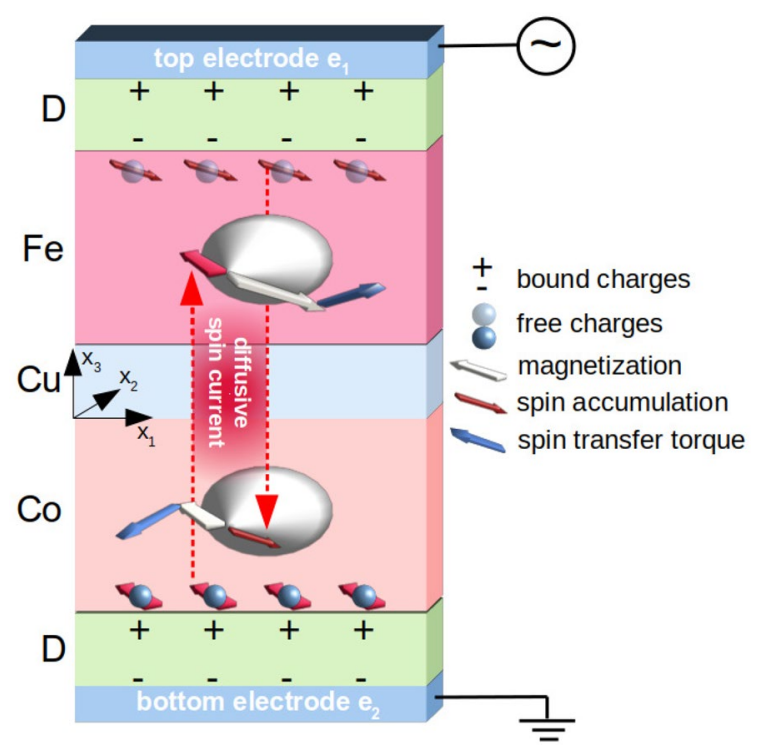

Figure 1. The principle of operation of magnetoelectric cell subjected to the ac voltage. The spin-dependent surface screening at the Fe/dielectric and $\mathrm{Co}$ /dielectric interfaces generates the spin accumulation which diffuses through the $\mathrm{Fe} / \mathrm{Cu} / \mathrm{Co}$ layers and exerts the spin-transfer torque on the local magnetization.

spin-dependent ${ }^{25-27}$, the dynamic spin-dependent potential produces nonequilibrium spin density at the interfaces, as we have demonstrated in Ref. ${ }^{13}$. Importantly, the strength of the effect depends on the magnetoelectric constant $\gamma_{s}$. The spin accumulation is polarized along the local, precessing magnetic moment at the interface with the dielectric, and it diffuses between the layers. If the magnetization of the second layer is non-collinear to that spin accumulation (i.e., to the magnetic moment at the surface of the first layer), it exerts a spin-transfer torque. From this, it arises that the spin-transfer torque depends on the profile of the dynamic magnetization through the bilayer thickness, i.e., the spin-wave mode profile.

To describe the coupled charge-spin-magnetization dynamics of the system and demonstrate usefulness of the MEC we solve numerically the set of differential equations for the bound $\left(n_{b}\right)$ and free $\left(n_{f}\right)$ charge densities, spin accumulation $\vec{s}$ and corresponding currents: displacement $\left(J_{d}\right)$, charge $(J)$ and spin $\left(J_{s}\right)$ currents, electric potential $V$ and magnetization $\vec{m}$. Although we implemented both spin-dependent surface screening and spin-dependent conductivity as sources of the spin accumulation in the model, the latter is neglible since interface effects play dominant role in the considered system. For the interaction between spin accumulation and magnetization at interfaces we applied the continuous approach for the spin-transfer torque ${ }^{28,29}$.

\section{The model}

The coupled charge-spin-magnetization dynamics driven by the ac voltage is considered on the base of the diffusive model ${ }^{28-31}$. The free charge density current $J_{f}$ is described in the metal layers by the equation:

$$
\vec{J}_{f}=\sigma \vec{E}-D \nabla n_{f}+\beta D \frac{e}{\mu_{B}}(\nabla \vec{s}) \hat{m}
$$

which includes contributions from electron drift driven by electric field $\vec{E}$, diffusion driven by the gradient of free charge density $n_{f}$ and a diffusive spin polarisation representing spin conductivity contribution, respectively. Here, $\sigma$ is conductivity, $D$ is diffusion constant, $\beta$ is spin asymmetry coefficient, $\mu_{B}$ is Bohr magneton and $e$ is a charge of electron. Versor $\hat{m}$ points along magnetization direction and in the linear regime it is $\hat{m}=\vec{m} / M_{s}=\left(m_{1} / M_{s}, 1, m_{3} / M_{s}\right)$.

In dielectric layers the displacement current is described by the equation:

$$
\vec{J}_{b}=\epsilon_{0} \epsilon_{r} \frac{\partial \vec{E}}{\partial t}
$$

where $\epsilon_{0} \epsilon_{r}$ is permittivity of the material. The conservation of free $(i=f)$ and bound $(i=b)$ charge density $n_{i}$ is described by the continuity equation:

$$
\frac{\partial n_{i}}{\partial t}=-\nabla \cdot \vec{J}_{i}
$$

Electric potential $V$ is given by the Gauss Law:

$$
\begin{aligned}
\epsilon_{0} \Delta V & =n_{f}+n_{b}, \\
\vec{E} & =-\nabla V
\end{aligned}
$$


with the boundary conditions at the electrodes:

$$
\begin{aligned}
& \left.V\right|_{x=e 1}=U \sin \left(2 \pi f_{U} t\right), \\
& \left.V\right|_{x=e 2}=0,
\end{aligned}
$$

where $f_{U}$ is the frequency of the oscillating voltage $U$.

The spin current described by the second rank tensor $\mathbf{J}_{\mathbf{s}}$ in the ferromagnetic material is modeled by the equation $^{31}$ :

$$
\begin{aligned}
\mathbf{J}_{s}= & -D \nabla \vec{s}-\beta \frac{\mu_{B}}{e}\left(\sigma \vec{E}-D \nabla n_{f}\right) \otimes \hat{m} \\
& -\frac{\tau}{\tau_{J}} \mathbf{J}_{\mathbf{s}} \times \hat{m}-\frac{\tau}{\tau_{\perp}} \hat{m} \times \mathbf{J}_{\mathbf{s}} \times \hat{m},
\end{aligned}
$$

which describes spin current driven by gradient of the spin accumulation $\vec{s}$ (diffusion), the spin-conductivity term. The last two terms (see the description of the constants below) are the ballistic corrections to the spintransfer torque in the diffusive model ${ }^{29}$.

The continuity equation for the spin accumulation $\vec{s}$ is:

$$
\frac{\partial \vec{s}}{\partial t}=-\nabla \cdot \mathbf{J}_{\mathbf{s}}-m_{0} \frac{\partial \vec{m}}{\partial t}-\frac{\vec{s}}{T_{1}}+\vec{\tau}_{\mathrm{STT}}+\vec{f}_{S},
$$

which describes the rate of change of $\vec{s}$ due to gradient of the spin current, spin pumping of magnitude $m_{0}{ }^{32,33}$ and the spin-flip relaxation with the characteristic time $T_{1}$. The spin-transfer torque $\vec{\tau}_{\text {STT }}$ is given by:

$$
\vec{\tau}_{\mathrm{STT}}=\tau_{J}^{-1} \hat{m} \times \vec{s}+\tau_{\perp}^{-1} \hat{m} \times \hat{m} \times \vec{s},
$$

and it is characterized by spin precession length $\lambda_{J}=2 \pi \sqrt{3} v_{F} \tau_{J}$ and spin coherence length $\lambda_{\perp}=\sqrt{3} v_{F} \tau_{\perp}$ where $v_{F}=1570 \mathrm{~nm} / \mathrm{ps}$ is the velocity of an electron at the Fermi level. The source term $\vec{f}_{S}$ from spin-dependent surface screening is ${ }^{13}$ :

$$
\vec{f}_{S}=\gamma_{S} \frac{\mu_{B}}{e} \frac{\partial n_{f}}{\partial t} \hat{m}
$$

The magnetization dynamics is given by the Landau-Lifshitz-Gilbert equation with spin-transfer torque term:

$$
\frac{\partial \vec{m}}{\partial t}=-\gamma \mu_{0} \vec{m} \times \vec{H}_{e f f}+\frac{\alpha}{M_{s}} \vec{m} \times \frac{\partial \vec{m}}{\partial t}-\vec{\tau}_{S T T},
$$

where $\gamma=176 \mathrm{GHz} / \mathrm{T}$ is gyromagnetic constant, $\mu_{0}$ is magnetic susceptibility and $\alpha$ is Gilbert damping constant.

The effective field $\vec{H}_{\text {eff }}$ contains exchange, dipolar and Zeeman interactions:

$$
\vec{H}_{e f f}=\frac{2 A}{\mu_{0} M_{s}^{2}} \Delta \vec{m}-\nabla \phi+H_{0} \hat{x}_{2}
$$

where $H_{0}$ is the external magnetic field applied along the $\hat{x}_{2}$ direction. The magnetic potential $\phi$ is given from the Maxwell equation:

$$
\Delta \phi=\nabla \cdot \vec{m} .
$$

Equations (1)-(12) are solved numerically by finite element method in Comsol Multiphysics with timevarying voltage applied to the MEC electrodes. The equations are implemented with the currents defined as a flux, thus assuring their continuity at interfaces and zero value at the outer boundaries.

The relative permittivity is $\epsilon_{r}=120$ for dielectric layer and $\epsilon_{r}=1$ for metals. We assume the conductivity of metals $\sigma=1.2 \times 10^{7} \mathrm{~S} / \mathrm{m}$ and the diffusion constant $D=4 \times 10^{-3} \mathrm{~m}^{2} / \mathrm{s}^{31}$. The spin asymmetry coefficient is $\beta=0.5^{31}$, the spin-flip relaxation time is $T_{1}=0.9 \mathrm{ps}^{31}$. This corresponds to the spin relaxation length of $60 \mathrm{~nm}$, i.e., much larger than electron mean free path, satisfying diffusive limit ${ }^{30,34,35}$. The magnitudes of spin pumping are $m_{0}=0.17$ for Co and $m_{0}=0.22$ for Fe. The characteristic times for the spin-transfer torques are $\tau_{J}=0.3 \mathrm{fs}, \tau_{\perp}=1.2 \mathrm{fs}$ and $\tau_{J}=0.2 \mathrm{fs}, \tau_{\perp}=4.4 \mathrm{fs}$ for Co and Fe, respectively ${ }^{29}$. The magnetoelectric coefficient calculated from Eq. (10) in Ref. ${ }^{13}$ is $\gamma_{S}=-0.25$ for Co and $\gamma_{S}=0.36$ for Fe. The exchange constants were taken $A=20 \mathrm{pJ} / \mathrm{m}$ both for Co and Fe, Gilbert damping $\alpha_{\mathrm{Co}}=0.005, \alpha_{\mathrm{Fe}}=0.002$ and the saturation magnetizations $M_{\mathrm{s}, \mathrm{Co}}=1 \mathrm{MA} / \mathrm{m}$ and $M_{\mathrm{s}, \mathrm{Fe}}=1.7 \mathrm{MA} / \mathrm{m}$. The external magnetic field straength is $H_{0}=10 \mathrm{kA} / \mathrm{m}$.

In the following part of the paper, we briefly discuss the dispersion relation and phase profiles of the spin-wave modes in a dipolarly coupled ferromagnetic Fe/Co bilayers which we use in the magnetoelectric cell. We consider magnetization dynamics in the Damon-Eshbach geometry, i.e., the saturation magnetization is perpendicular to the wave vector $k$ and lies in the plane of the films. This geometry, in principle, assures higher group velocity of the wave with respect to the backward volume geometry and effectively lower damping with respect to the forward volume geometry. We neglect in our model surface anisotropies that may play a role in thin-film systems but will not influence the effect of spin-wave amplification qualitatively.

Next, the magnetization dynamics for the case of uniform magnetization precession (wave vector $k=0$ ) is presented with the ac voltage applied to the MEC. Then, the practical realization of the modulation and amplification of the propagating spin wave is proposed and the simulation results for the optimized MEC geometry 


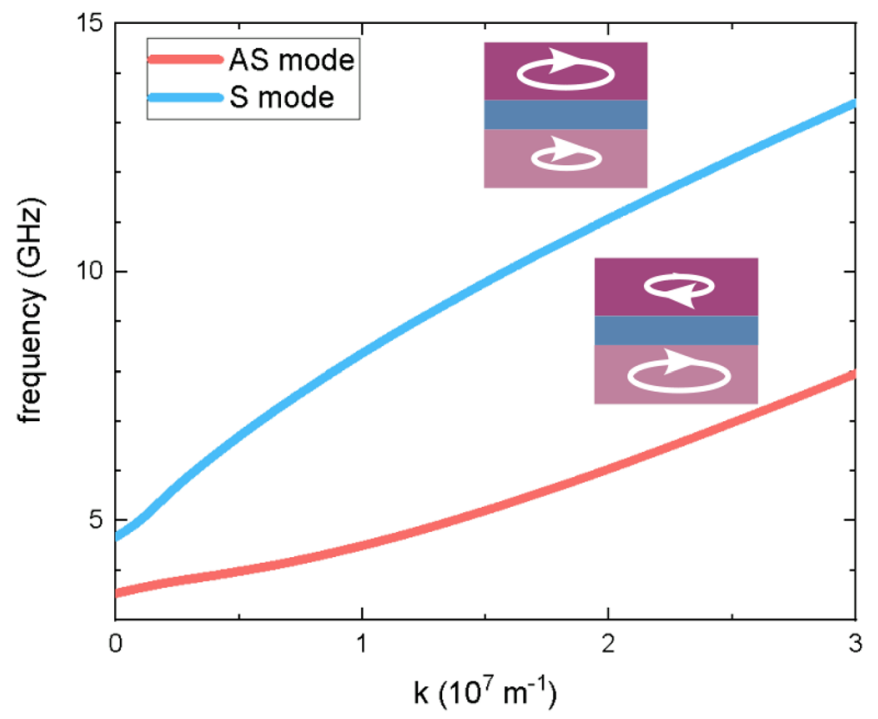

Figure 2. The spin-wave dispersion relation for the $\mathrm{Fe} / \mathrm{Cu} / \mathrm{Co}$ multilayer in the Damon-Eshbach configuration. The insets show relative phase and amplitude of the magnetization precession for the antisymmetric (AS) and symmetric (S) modes in Fe and Co layers.

presented. In the discussion section we analyze the symmetry, material and geometric requirements to get the effective spin-wave modulation in a thin film with MEC.

\section{Results}

In all the results shown below, unless otherwise stated, we applied ac voltage of amplitude $U=10 \mathrm{~V}$ and frequency $f_{U}=200 \mathrm{MHz}$ to the MEC electrodes. This frequency is below resonant frequency of the magnetization in the considered system and it produces enough spin accumulation at FM/D interface to affect magnetization dynamics. The amplitude of ac charge current is $J=10^{5} \mathrm{~A} / \mathrm{cm}^{2}$. The thickness of each layer of the $\mathrm{D} / \mathrm{Fe} / \mathrm{Cu} /$ $\mathrm{Co} / \mathrm{D}$ stack is assumed to be $2 \mathrm{~nm}$, except $\mathrm{Cu}$ which is $5 \mathrm{~nm}$ thick.

Spin-wave dispersion relation of the bilayer system. The calculated dispersion relation of the two spin-wave modes of the lowest frequency in the $\mathrm{Fe} / \mathrm{Cu} / \mathrm{Co}$ system is shown in Fig. 2. The low-frequency mode (red line) with the amplitude concentrated mainly in Co is an antisymmetric mode (AS), i.e., the phase of magnetization precession between layers is shifted by $\pi$ (see insets in Fig. 2). The second mode of the higher frequency (blue line) with the amplitude concentrated mainly in $\mathrm{Fe}$ is a symmetric mode (S), i.e., with the in-phase magnetization precession in Co and Fe. Both modes have almost completely uniform amplitude through each layer thickness. Note, that for $k=0$ the modes in Fe and Co are independent since there is no dipolar coupling between the layers in this case ${ }^{36}$. The higher-order modes are in the teraherz range.

Spin wave amplification. We will first demonstrate the effect of the ac voltage on the uniform precession of the magnetization $(k=0)$. The magnetization at the starting time $(t=0)$ is deviated from its equilibrium direction $x_{2}$ in the Co layer by taking initial value of the dynamic magnetization $m_{1}=10 \mathrm{~A} / \mathrm{m}$. Then, the magnetization precesses freely with the frequency $3.4 \mathrm{GHz}$, i.e., frequency of the antisymmetric mode. If we do not apply any voltage, the precession amplitude decays exponentially with time due to the Gilbert damping as shown with black lines in Fig. 3.

With the ac voltage applied to the MEC, the precession of the magnetization in Co is enhanced in the first half of the voltage and current cycle, but it is strongly damped in the second half of this cycle (see, the red lines in Fig. 3). After one cycle of the voltage (5 ns) the amplitude of precession is exactly the same as in the case without the voltage. Therefore, the average impact of the voltage on the spin wave is zero. Since the Fe and Co layers are decoupled for $k=0$ and they have different ferromagnetic resonance (FMR) frequencies, the STT does not induce coherent precession in Fe layer.

With the propagating spin wave we can map the temporal modulation of the magnetization dynamics induced by the ac voltage into the spatiotemporal distribution of the spin-wave amplitude. For this purpose the MEC of finite length is chosen with the width $w_{\text {MEC }}=v / f_{U}$, estimated with respect to the spin-wave group velocity $v$ and the voltage frequency $f_{U}$. For that width, the wavefront entering MEC area at the time when the voltage starts to enhance the magnetization dynamics, leaves MEC area when the voltage stops to enhance, i.e., after half of the voltage period. Therefore, for this MEC width the spin wave should be maximally enhanced and we use this width in the following simulations.

The system proposed for the spin-wave modulation and amplification is shown in Fig. 4. It consists of the extended Co thin film and the MEC of the finite width. Because of the mode symmetry which translates into the 


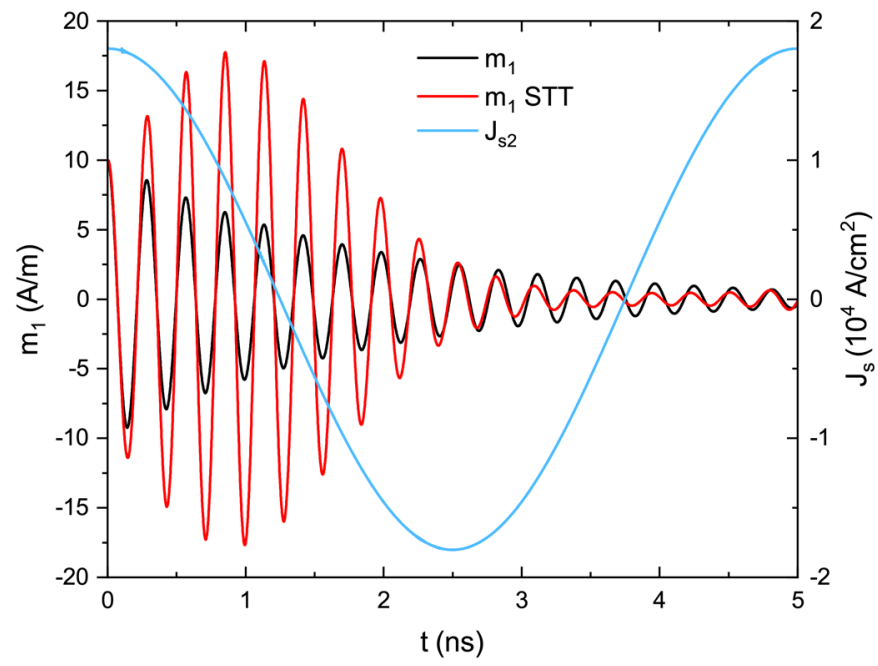

Figure 3. The time-dependence of the magnetization component $m_{1}$ (red line) and spin current $J_{s 2}$ (blue line) in Co in the MEC subjected to the ac voltage; black line shows $m_{1}$ dynamics in the absence of ac voltage.

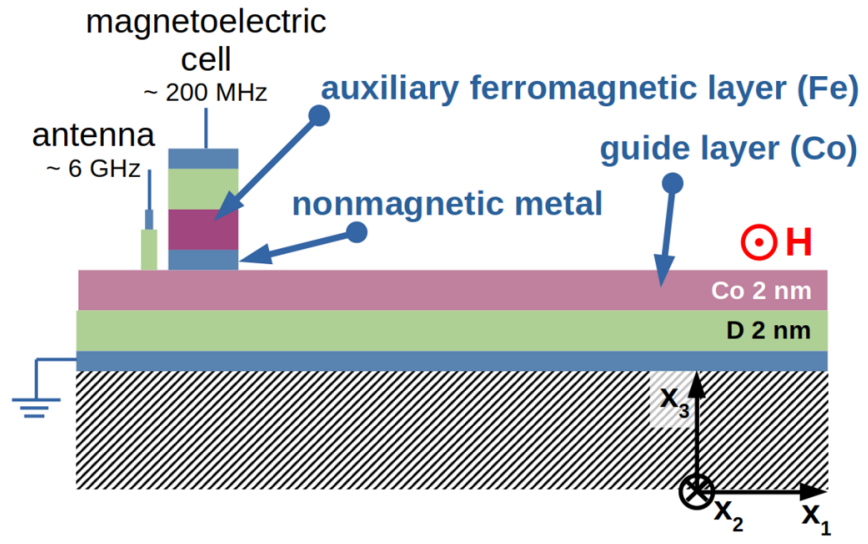

Figure 4. The spin-wave waveguide from Co thin film with the magnetoelectric cell for amplification and amplitude modulation of the spin waves.

STT direction (discussed in the next section), only the asymmetric mode (related to the Co layer) is affected by STT. Therefore, we have chosen Co layer as a guide layer for the spin wave, while Fe acts as an auxiliary layer in MEC. The width of MEC $w_{\text {MEC }}=2.25 \mu \mathrm{m}$ is optimized for the group velocity of the asymmetric spin-wave mode at the frequency of $6 \mathrm{GHz}$. The antenna of the width of $150 \mathrm{~nm}$ is placed $1 \mu \mathrm{m}$ before MEC and it excites spin wave with the monochromatic microwave magnetic field of the amplitude of $1 \mathrm{~A} / \mathrm{m}$, homogeneous in the area of the antenna. We introduced artificial damping at the left and right edges of Co film to avoid unnecessary reflections.

Figure 5 shows the results of the numerical time-dependent simulations. The plane spin wave at frequency $6 \mathrm{GHz}$ is continuously excited by the antenna and it propagates to the right entering MEC. Under the MEC the spin wave is subjected to the STT from the spin accumulation generated by a dynamic spin-dependent surface screening. At the output of MEC we obtain a spin wave with modulated amplitude. The inset in Fig. 5 shows the spin-wave dynamic component $m_{1}$ at a fixed point behind MEC ( $2 \mu \mathrm{m}$ after MEC) in dependence on time. The signal is periodically modulated: amplified and suppressed with the frequency of the ac voltage $f_{U}$ applied to MEC. When comparing the maximum amplitude of the spin wave at the output of MEC with the amplitude of the spin wave at the same position but in the absence of the ac voltage, we found the gain of the spin-wave amplitude reaching 24 .

Interestingly, the gain introduced by the MEC increases exponentially with the amplitude of the voltage as is shown in Fig. 6a. Further, we check how the MEC of the fixed width and for the fixed voltage frequency influences the spin waves at different frequencies (Fig. 6b). As the MEC width and the voltage frequency are optimized for the group velocity of the spin wave at $6 \mathrm{GHz}$, the gain decreases for other frequencies. However, since the group velocity does not vary severely in this range of frequency, the gain remains at significant level, it is above 15 in a broad range of frequencies exceeding $5 \mathrm{GHz}$. The points achieved from the simulation in Fig. $6 \mathrm{~b}$ are compared to the dependence calculated based on the spin-wave group velocity $v$ obtained from the dispersion relation in 


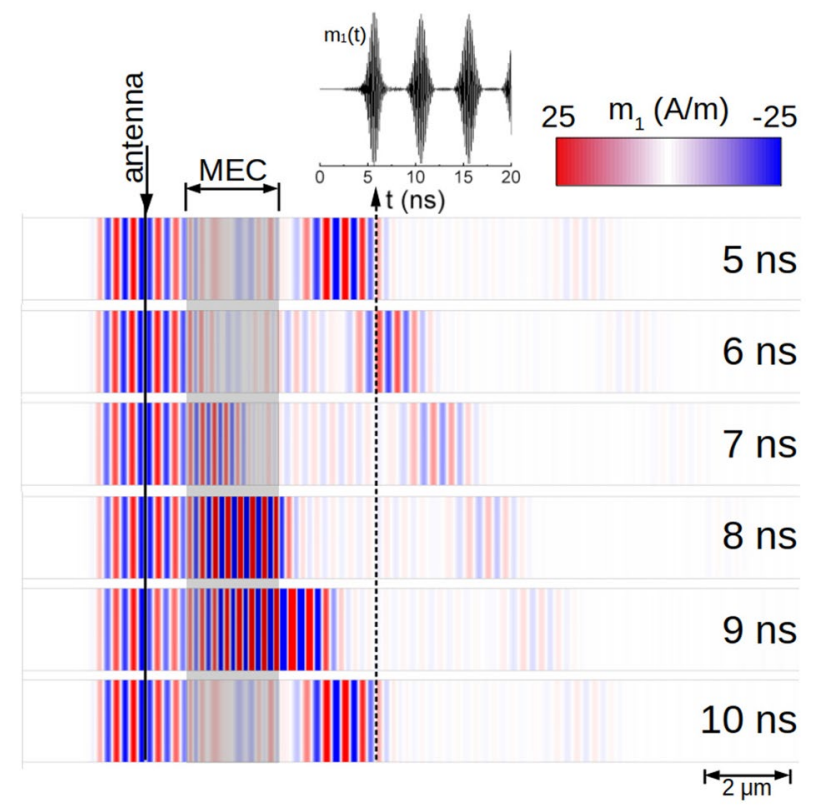

Figure 5. Magnetization component $m_{1}$ of the spin-wave AS mode at $6 \mathrm{GHz}$ in Co which passes MEC for different points in time. The inset shows the time dependence of $m_{1}$ at the fixed point in Co film after the output of MEC.
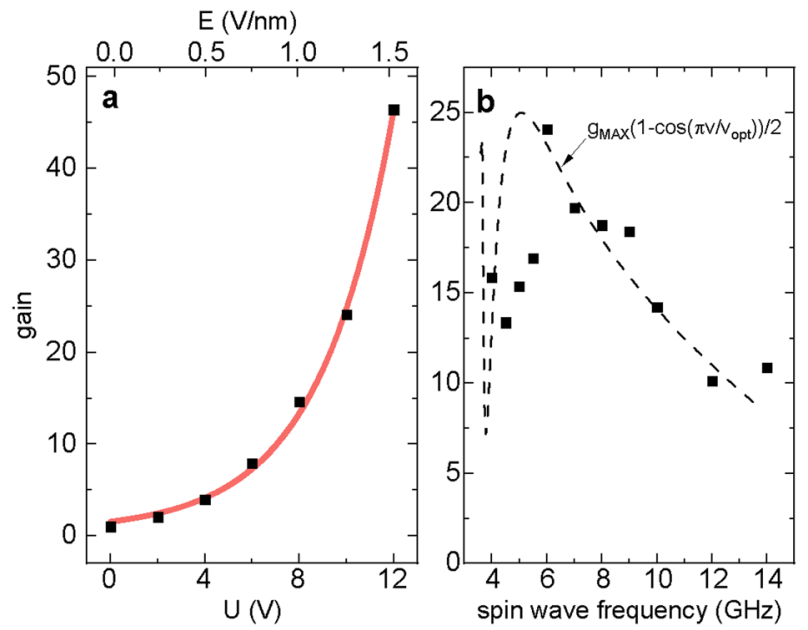

Figure 6. (a) The dependence of the maximum gain of the spin-wave amplitude on the amplitude of the ac voltage in MEC; (b) the dependence of the gain on the spin-wave frequency passing MEC. Black points are the results of simulations, while the black dashed curve in $(\mathbf{b})$ is obtained from the group velocity analysis.

Fig. 2. Here, $v_{\mathrm{opt}}$ is the group velocity for the spin wave of $6 \mathrm{GHz}$ and $g_{\mathrm{MAX}}=24$ is the maximum gain obtained in simulations. Going from high frequencies, the gain reaches maximum at $6 \mathrm{GHz}$ (for $v_{\text {opt }}$ ), suddenly drops at lower frequencies and increases again at the lowest frequencies. This is because the group velocity for the antisymmetric mode (see the red line in Fig. 2) increases for $k \approx 0$. While the theoretical line fits well for higher frequencies, some differencies are visible at lower frequencies, which is the result of the reflections at the MEC edges.

\section{Discussion}

To explain obtained results we will describe the influence of the voltage-driven STT onto magnetization dynamics in a bilayer system for the propagating spin wave. Fig. 7 shows the precession cone of the magnetization in $\mathrm{Fe}$ (left) and Co (right) layers for the mode S (up) and mode AS (bottom) and the magnetization direction at a given time (black arrows). In the further part, we will refer to the situation at a particular moment of the voltage/ current dynamics. As described in the introduction, the dynamic spin-dependent charge screening produces spin accumulation at the ferromagnetic-dielectric interfaces. The spin accumulation is polarized along the local, 


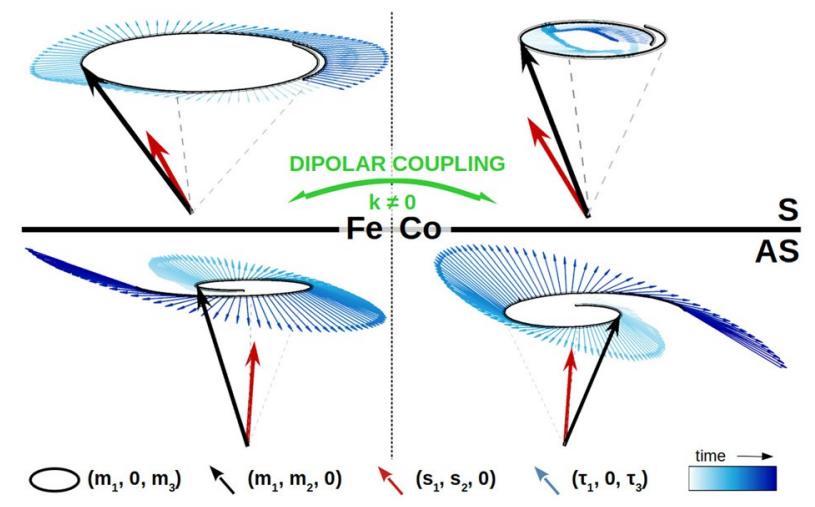

Figure 7. The precession cone of the magnetization in Fe (left) and Co (right) layer for the symmteric mode $S$ (up) and antisymmetric mode AS (bottom), the spin accumulation and generated spin-transfer torques. The precession trajectory and spin-transfer torques are taken from numerical simulations. The spin transfer torques are averaged through each layer thickness.

precessing magnetic moment at the interface, i.e., $\vec{s}_{\mathrm{Fe}} \propto \vec{m}_{\mathrm{Fe}}$ and $\vec{s}_{\mathrm{Co}} \propto \vec{m}_{\mathrm{Co}}$. The spin accumulation gradient $\delta \vec{s}=\vec{s}_{\mathrm{Fe}}-\vec{s}_{\mathrm{Co}}$ is quickly compensated as a result of the diffusive transport between the layers. The resultant spin accumulation $\vec{s}=\left(\vec{s}_{\mathrm{Fe}}+\vec{s}_{\mathrm{Co}}\right) / 2$ shown in Fig. 7 by red arrows decays now further due to the STT interaction with the magnetization and the spin-flip relaxation.

Because the precession of the magnetization has different amplitude in Co and Fe layers for the mode $S$ (upper part of Fig. 7), the spin accumulation $\vec{s}$ points inside the precession cone in Fe and it points outside the precession cone in the Co. As a consequence, the spin-transfer torque acts in the opposite direction in both layers. Since the magnetization dynamics is coupled by the dipolar fields, the overall influence of the STT on the magnetization dynamics on the mode $\mathrm{S}$ is suppressed.

The bottom part of Fig. 7 shows magnetization precession for the AS mode. The spin accumulation direction points in a manner similar to the case above with respect to the magnetization direction at a given time. The STT directions are also opposite between layers. However, as the precession in the layers are out-of-phase (the $\pi$ phase shift) in the AS mode, the STT direction points outside the precession cone in both layers. Therefore, the magnetization precession is enhanced both in Fe and Co. The dipolar fields acting between layers lead to the enhancement of the AS mode. In the opposite phase of the voltage change the mode is effectively damped, since the sign of the spin accumulation and STT is opposite.

Now we can analise the case for or $k=0$ more deeply. There, STT modulates the amplitude of the magnetization dynamics for both modes: FMR in the Fe layer and FMR in the Co layer. This is because for $k=0$ the layers do not interact via dipolar fields, so the dynamics in the Fe and Co layers is coupled solely by STT. STT acts for the FMR mode in Fe in the same way as on the S mode shown in Fig. 7, i.e., it enhances precession in one layer and it reduces precession in the other layer. However, the reduction of the precession in one layer does not influence the enhancement in the other layer, because the dynamics is dipolarly decoupled. For the FMR mode in Co the STT acts to enhance the precession in both layers. However, the enhancement of precession in Fe does not strengthen precession in Co through dipolar field, so the amplification of the FMR mode is much weaker as compared to the AS mode at $k \neq 0$. Moreover, the dynamics driven by the STT onto Fe is not coherent, since the resonance frequencies of the layers are different.

To maximize the effect of the voltage-driven amplification, we have chosen ferromagnetic materials with the opposite sign of the magnetoelectric constant $\left(\gamma_{s}=-0.25\right.$ for Co and $\gamma_{s}=0.36$ for Fe). With this selection, for the opposite charge densities which accumulate at the $\mathrm{D} / \mathrm{Fe}$ and $\mathrm{Co} / \mathrm{D}$ interfaces, the induced spin accumulation is parallel (or antiparallel) to the magnetization in both layers. The same sign of the spin accumulation component along the direction of the magnetization saturation $\left(x_{2}\right)$ in the layers blocks its depletion due to the diffusion between layers (small spin accumulation gradient $\delta \vec{s}$ ) and thus it assures its high value in the system which is essential for high value of STT. Alternatively, one can use the bilayer of the same material but with the antiparallel magnetization alignment ${ }^{37}$. In this case, both modes that exists in the system are alternated by STT. However, STT acts congruently between the layers only at sectional magnetization positions in the precession cone for the antiparallel configuration. Therefore, the modulation effect is much weaker.

To obtain high density of screening charges and spin accumulation at the dielectric interface, the high dielectric constant is necessary. From this point of view, ferroelectric capacitors should be considered. However, the complexity of ferroelectric crystal structure makes it difficult to obtain ferroelectrics of the quality high enough to prevent leakage currents ${ }^{38}$. Then, the only dielectric films used experimentally so far to stand the electric field of the order of $\mathrm{V} / \mathrm{nm}$ are $\mathrm{MgO}$ and $\mathrm{HfO}_{2}{ }^{39-41}$. So, although there exist materials that stand high electric fields or posses high dielectric constant, nowadays, it is challenging to find a perfect candidate that fulfill both criteria simultaneously. 


\section{Summary}

We have shown numerically that the voltage-driven spin accumulation in magnetoelectric cell exerts the spintransfer torque on the magnetization in a bilayer system. The maximum induced charge current in the MEC is of the order of $10^{5} \mathrm{~A} / \mathrm{cm}^{2}$. That is an order of magnitude less than the current needed for control of the attenuation by spin Hall effect shown by An et al. ${ }^{14}$ and 4 orders of magnitude less than control of the attenuation by spin-dependent conductivity with in-plane current shown by Seo et al. ${ }^{12}$. Depending on the mode symmetry, STT driven by spin-dependent surface screening may lead or may not lead to the modulation of the spin-wave amplitude. The investigated magnetoelectric cell, although its width is optimized for the particular spin-wave velocity and voltage frequency, is able to modulate spin waves in relatively broad range of frequencies from FMR to several GHz. However, it will not have, contrary to other reported amplification techniques based on spin-orbit torques, the influence on the incoherent noise.

Since ac voltage is required to obtain the spin accumulation in the system, the presented mechanism is not compatible for the continuous spin-wave amplification. However, it may be useful for other applications, e.g., for the modulation of the spin-wave amplitude, amplification of short impulses or when the effect will be used for two parallel spin-wave conduits operating on the split signal with respective microwave fields delayed by $\pi$. The generation of nonequilibrium spin density through dynamic spin-dependent surface screening in the proposed magnetoelectric heterostructure allows to reduce the thickness of fixed magnetization layer used in conventional spin valve to a few nanometers, thus the proposed effect can significantly contribute to miniaturization of the spintronic and magnonic devices.

Received: 23 April 2021; Accepted: 12 July 2021

Published online: 03 August 2021

\section{References}

1. Chumak, A. V., Vasyuchka, V. I., Serga, A. A. \& Hillebrands, B. Magnon spintronics. Nat. Phys. 11, 453 (2015).

2. Smith, K. R., Vasyuchka, V. I., Wu, M., Melkov, G. A. \& Patton, C. E. Cloning and trapping of magnetostatic spin-wave pulses by parametric pumping. Phys. Rev. B 76(5), 054412 (2007).

3. Brächer, T. et al. Mode selective parametric excitation of spin waves in a Ni 81Fe19 microstripe. Appl. Phys. Lett. 99(16), 162501 (2011).

4. Brächer, T. et al. Time- and power-dependent operation of a parametric spin-wave amplifier. Appl. Phys. Lett. 105(23), 232409 (2014).

5. Brächer, T. et al. Parallel parametric amplification of coherently excited propagating spin waves in a microscopic Ni81Fe19 waveguide. Appl. Phys. Lett. 104(20), 202408 (2014).

6. Balinskiy, M. et al. Magnetoelectric spin wave modulator based on synthetic multiferroic structure. Sci. Rep. 8(1), 10867 (2018).

7. Khitun, A., Nikonov, D. E. \& Wang, K. L. Magnetoelectric spin wave amplifier for spin wave logic circuits. J. Appl. Phys. 106(12), 123909 (2009).

8. Cherepov, S. et al. Electric-field-induced spin wave generation using multiferroic magnetoelectric cells. Appl. Phys. Lett. 104(8), 082403 (2014).

9. Chowdhury, P., Dhagat, P. \& Jander, A. Parametric amplification of spin waves using acoustic waves. IEEE Trans. Magnet. 51(11), 1300904 (2015).

10. Graczyk, P. \& Krawczyk, M. Coupled mode theory for the acoustic wave and spin wave interaction in the magphonic crystals: Propagating magnetoelastic waves. Phys. Rev. B 96, 024407 (2017).

11. Nikitchenko, A. I. \& Pertsev, N. A. Spin injection and pumping generated by a direct current flowing through a magnetic tunnel junction. Phys. Rev. B 99(22), 224426 (2019).

12. Seo, S. M., Lee, K. J., Yang, H. \& Ono, T. Current-induced control of spin-wave attenuation. Phys. Rev. Lett. 102(14), 147202 (2009).

13. Graczyk, P. \& Krawczyk, M. Spin-polarized currents driven by spin-dependent surface screening. Phys. Rev. B 100, 195415 (2019).

14. An, K. et al. Control of propagating spin waves via spin transfer torque in a metallic bilayer waveguide. Phys. Rev. B Condensed Matter Mater. Phys. 89(14), 140405(R) (2014).

15. Duan, Z. et al. Spin-wave modes in permalloy/platinum wires and tuning of the mode damping by spin Hall current. Phys. Rev. B 90(2), $024427(2014)$.

16. Hamadeh, A. et al. Full control of the spin-wave damping in a magnetic insulator using spin-orbit torque. Phys. Rev. Lett. 113(19), 197203 (2014).

17. Padrón-Hernández, E., Azevedo, A. \& Rezende, S. M. Amplification of spin waves in yttrium iron garnet films through the spin Hall effect. Appl. Phys. Lett. 99(19), 192511 (2011).

18. Wang, Z., Sun, Y., Wu, M., Tiberkevich, V. \& Slavin, A. Control of spin waves in a thin film ferromagnetic insulator through interfacial spin scattering. Phys. Rev. Lett. 107(14), 146602 (2011).

19. Jungfleisch, M. B. et al. Interface-driven spin-torque ferromagnetic resonance by Rashba coupling at the interface between nonmagnetic materials. Phys. Rev. B 93(22), 224419 (2016).

20. Amin, V. P. \& Stiles, M. D. Spin transport at interfaces with spin-orbit coupling: Formalism. Phys. Rev. B 94(10), 104419 (2016).

21. Amin, V. P., Zemen, J. \& Stiles, M. D. Interface-generated spin currents. Phys. Rev. Lett. 121(13), 136805 (2018).

22. Demidov, V. E. et al. Magnetic nano-oscillator driven by pure spin current. Nat. Mater. 11(12), 1028-1031 (2012).

23. Fan, Y. \& Wang, K. L. Spintronics based on topological insulators. Spin 06(02), 1640001 (2016).

24. Navabi, A. et al. Control of spin-wave damping in YIG using spin currents from topological insulators. Phys. Rev. Appl. 11(3), 034046 (2019).

25. Zhang, S. Spin-dependent surface screening in ferromagnets and magnetic tunnel junctions. Phys. Rev. Lett. 83(3), 640-643 (1999).

26. Zhuravlev, M. Y., Maekawa, S. \& Tsymbal, E. Y. Effect of spin-dependent screening on tunneling electroresistance and tunneling magnetoresistance in multiferroic tunnel junctions. Phys. Rev. B 81(10), 104419 (2010).

27. Niranjan, M. K., Burton, J. D., Velev, J. P., Jaswal, S. S. \& Tsymbal, E. Y. Magnetoelectric effect at the $\mathrm{SrRuO}_{3} / \mathrm{BaTiO}_{3}(001)$ interface: An ab initio study. Appl. Phys. Lett. 95, 052501 (2009).

28. Lepadatu, S. Unified treatment of spin torques using a coupled magnetisation dynamics and three-dimensional spin current solver. Sci. Rep. 7(1), 12937 (2017).

29. Petitjean, C., Luc, D. \& Waintal, X. Unified drift-diffusion theory for transverse spin currents in spin valves, domain walls, and other textured magnets. Phys. Rev. Lett. 109(11), 117204 (2012).

30. Valet, T. \& Fert, A. Theory of the perpendicular magnetoresistance in magnetic multilayers. Phys. Rev. B 48(10), 7099-7113 (1993).

31. Zhu, Y.-H., Hillebrands, B. \& Schneider, H. C. Signal propagation in time-dependent spin transport. Phys. Rev. B 78, 054429 (2008).

32. Takahashi, S. Physical principles of spin pumping. in Handbook of Spintronics (ed. Xu, T.) 1445-1480 (Springer, 2016). 
33. Zhang, S. \& Li, Z. Roles of nonequilibrium conduction electrons on the magnetization dynamics of ferromagnets. Phys. Rev. Lett. 93(12), 127204 (2004).

34. Penn, D. R. \& Stiles, M. D. Spin transport for spin diffusion lengths comparable to mean free paths. Phys. Rev. B 72(21), 212410 (2005).

35. Gmitra, M. \& Barnaś, J. Correlation of the angular dependence of spin-transfer torque and giant magnetoresistance in the limit of diffusive transport in spin valves. Phys. Rev. B 79(1), 012403 (2009).

36. Graczyk, P., Zelent, M. \& Krawczyk, M. Co- and contra-directional vertical coupling between ferromagnetic layers with grating for short-wavelength spin wave generation. New J. Phys. 20, 053201 (2018).

37. Graczyk, P. et al. Magnonic band gap and mode hybridization in continuous permalloy films induced by vertical dynamic coupling with an array of permalloy ellipses. Phys. Rev. B 98, 174420 (2018).

38. Graczyk, P. \& Coy, E. Single-phase multiferroics. in Nanostructured Multiferroics (eds Balakrishnan, R. \& Visakh, P. M.) 38 (Wiley, 2021).

39. Matsukura, F., Tokura, Y. \& Ohno, H. Control of magnetism by electric fields. Nat. Nanotechnol. 10(3), 209-220 (2015).

40. Chen, Y.-J. et al. Parametric resonance of magnetization excited by electric field. Nano Lett. 17, 572-577 (2017).

41. Wang, W.-G., Li, M., Hageman, S. \& Chien, C. Electric-field-assisted switching in magnetic tunnel junctions. Nat. Mater. 11, 64 (2011).

\section{Acknowledgements}

The study has received financial support from the National Science Centre of Poland under Grant 2018/28/C/ ST3/00052.

\section{Author contributions}

Manuscript preparation: P.G. and M.K. Original research idea: P.G. and M.K. Model preparation and numerical simulations: P.G. All authors reviewed the manuscript.

\section{Competing interests}

The authors declare no competing interests.

Additional information

Correspondence and requests for materials should be addressed to P.G.

Reprints and permissions information is available at www.nature.com/reprints.

Publisher's note Springer Nature remains neutral with regard to jurisdictional claims in published maps and institutional affiliations.

(c) (i) Open Access This article is licensed under a Creative Commons Attribution 4.0 International cc) License, which permits use, sharing, adaptation, distribution and reproduction in any medium or format, as long as you give appropriate credit to the original author(s) and the source, provide a link to the Creative Commons licence, and indicate if changes were made. The images or other third party material in this article are included in the article's Creative Commons licence, unless indicated otherwise in a credit line to the material. If material is not included in the article's Creative Commons licence and your intended use is not permitted by statutory regulation or exceeds the permitted use, you will need to obtain permission directly from the copyright holder. To view a copy of this licence, visit http://creativecommons.org/licenses/by/4.0/.

(c) The Author(s) 2021 